\title{
An Unprecedented Stimuli Controlled Single-crystal Reversible Phase Transition of a Metal-Organic Framework and its Application to a Novel Method of Guest Encapsulation
}

Fangchang Tan, Ana López-Periago, Mark E. Light, Jordi Cirera, Eliseo Ruiz, Alejandro Borrás, Francesc Teixidor, Clara Viñas, Concepción Domingo, José Giner Planas*

F. Tan, Dr. A. López-Periago, Prof. F. Teixidor, Prof. C. Viñas, A. Borrás, Prof. C. Domingo, Dr. J. G. Planas

Institut de Ciència de Materials de Barcelona (ICMAB-CSIC), Campus UAB, 08193 Bellaterra, Spain

E-mail: jginerplanas@icmab.es

Dr. M. E. Light

Department of Chemistry, University of Southampton, Highfield, Southampton SO17 1BJ, UK

Dr. J. Cirera, Prof. E. Ruiz

Departament de Química Inorgànica i Orgànica and Institut de Recerca de Química Teòrica i Computacional, Universitat de Barcelona, Diagonal 645, 08028 Barcelona, Spain

Keywords: metal-organic frameworks, soft porous crystals, flexible porous materials, carboranes

The flexibility and unexpected dynamic behavior of a third generation MOF is described for the first time. The synthetic strategy is based on the flexibility and spherical shape of dipyridyl based carborane linkers that act as pillars between rigid $\mathrm{Co} / \mathrm{BTB}$ (BTB: 1,3,5benzenetricarboxylate) layers, providing a 3D porous structure (1). By manipulating the hostguest interactions between different solvents and the porous 3D structure of $\mathbf{1}$, a phase transition of the solid can be induced to generate a new, non-porous 2D structure (2) without any loss of the carborane linkers. However, the linkers are now coordinated to Co atoms in the same layer. The structural transformation was visualized by snapshots of the multi-step single-crystal-to-single-crystal transformation by single crystal and powder X-ray diffraction. Poor hydrogen bond acceptors such as $\mathrm{MeOH}, \mathrm{CHCl}_{3}$ or supercritical $\mathrm{CO}_{2}$ induce such a 3D to $2 \mathrm{D}$ transformation. Remarkably, the transformation is reversible and the $2 \mathrm{D}$ phase $\mathbf{2}$ is further converted back into 1 by heating in DMF. Intra-layer Co-N bond cleavage and inter- 
layer Co-N formation occur under such conditions, with the recovery of the initial porous 3D structure 1. The energy requirements involved in such processes have been investigated using periodic Density Functional Theory (DFT) calculations. As a proof of concept for potential applications, encapsulation of $\mathrm{C}_{60}$ has been achieved by trapping this molecule during the reversible $2 \mathrm{D}$ to $3 \mathrm{D}$ phase transition, whereas no adsorption was observed by straight solvent diffusion into the pores of the $3 \mathrm{D}$ phase.

So called "stimuli-responsive" or "smart" materials have the ability to go through conformational changes or phase transitions as a result of external chemical or physical stimuli.[1] Such responsiveness to specific stimuli or local environment is typical for biomolecules in nature but it is particularly difficult to achieve artificially. Such materials form the corner stone of developing intelligent technologies and are at the forefront of strategies addressing a number of global challenges.[2] Porous Coordination Polymers (CPs) or Metal-Organic Frameworks (MOFs) are a class of porous crystalline materials formed by the assembly of metal ions or metal clusters with different types of bridging organic linkers or ligands.[3] Third-generation MOFs, also known as "soft porous crystals" (SPCs) are defined as "porous solids that possess both a highly ordered network and structural transformability. They are bistable or multistable crystalline materials with long range structural ordering, a reversible transformability between states, and permanent porosity, at least in one crystal phase".[4]. These latter materials undergo a structural change, known as a "breathing" phenomenon, whereby the framework flexes in a reversible fashion in response to the changing nature and quantity of the adsorbed guest. However, flexibility often comes with the penalty of reduced stability, which limits their applications. Thus, the development of flexible yet stable third-generation MOFs depends on a better fundamental understanding of the conformational changes or phase transitions associated with such flexibility, opening the way to the rational design of novel "smart" or stimuli-responsive materials.[5] Despite the exciting 
application opportunities of these SPCs, e.g. in selective gas adsorption, chemical sensing or hazardous waste adsorption,[6] to date, there is no reported fully rational method for designing a breathing MOF. Framework flexibility is usually associated with flexible ligands, bond cleavage and regeneration, metal-metal interactions, supramolecular host-guest interactions, or a combination of a number of these.[4,5b-e, 7] Ligands in MOFs or CPs are usually built from aromatic fragments with planar structures (e.g, phenyl, pyridyl, porphirinyl).[5e, 8] Some degree of flexibility can be easily introduced by using flexible ligands, e.g., by adding aliphatic chains to the ligand structures or by substituting the planar $\pi$-systems by non-planar ones.[9] However, as mentioned above, flexibility often comes with the penalty of reduced stability. Collapsing of the structures is generally the rule, and reversible flexibility mostly a question of serendipity. In this investigation, we argue that with an appropriate design, the substitution of planar by spherical systems could influence the dynamic behavior of MOFs in the sense of avoiding or limiting the collapse of the structure. Spherical shaped linkers in 3D MOFs could help in stabilizing flexible structures. Different from classical rigid flat aromatic ligands' based MOFs, spherical based linkers can access to extensive conformational space by a combination of low-energy torsion of the substituents (e.g., aliphatic) and by the spherical core of the linker. Thus, a spherical ligand shape could diminish or even prevent the structure collapse simply by its globular nature or multiple supramolecular contacts, as opposed to the conventional planar nature of the ligands commonly employed. The idea of spherically shaped linkers avoiding collapse of the structure can also be understood like this: two layers will roll over each other if separated by spheres; whereas they will collapse if non-spherical columns are used (like in classical aromatic linkers). Suitable spherical systems for ligand syntheses are represented by $\mathrm{C}_{60}$ or dodecahedrane $\left(\mathrm{C}_{20} \mathrm{H}_{20}\right)$. These carbon-based molecules can be envisioned with various heteroatoms incorporated, but their synthesis is a challenging task.[10] In comparison, icosahedral boranes and carboranes $\left(\left[\mathrm{B}_{12} \mathrm{H}_{12}\right] 2-, 1, \mathrm{n}_{-} \mathrm{C}_{2} \mathrm{~B}_{10} \mathrm{H}_{12}(\mathrm{n}=2,7\right.$ or 12$\left.)\right)$ are an interesting class of commercially available and exceptionally stable 3D-aromatic[11] 
boron-rich clusters that possess material-favorable properties such as thermal and chemical stability and hydrophobicity.[12] Interestingly, such molecules can be easily functionalized by a wide range of known chemical transformations.[13] These icosahedral boron-based molecules can be visualized as hollow spheres. This fact has previously been exploited to provide carborane capped gold nanoparticles that exhibit redox-dependent solubility and phase transfer between water and nonpolar solvents[14] and also carborane linked quantum dots with unprecedented Kinetic Fluorescence Switching.[15] The packing of the spherical carborane moieties creates openings or channels that are crucial for such unprecedented properties. Herein we report a SPC containing a flexible carborane linker that reversibly responds to guest exchange through multi-step SCSC transformations, in which the synthesis of a crystalline 2D material is attained from a 3D framework and vice versa.

In pursuit of synthesizing water resistant architectures in general, and dynamic MOFs in particular, we have recently initiated the design of flexible carborane based ligands.[16] In this endeavor, we have recently reported a water stable SPC that behaves as a crystalline sponge for a variety of guests $\left[\mathrm{Co}(\mathrm{BTB})_{2}(\mathrm{mCB})_{2}\right] \cdot$ Guest $\quad(1 \supset$ Guest; BTB: 1,3,5benzenetricarboxylate, $\quad \mathrm{mCB}: \quad$ 1,7-bis $\{($ piridin-4-yl)methanol $\}-1,7-$ dicarba-closododecaborane; left column in Figure 1 and Figure S1 in the Supporting information).[16b] The available open space of this SPCs is not just a container for guest accommodation, but it demonstrates host-guest interactions between the framework and the guest molecules. Hence, either a contraction (in methanol $(\mathrm{MeOH})$ or chloroform $\left(\mathrm{CHCl}_{3}\right)$ ) or an expansion (in benzene, nitropropane, toluene or nitrobenzene) of the unit cell parameters of $\mathbf{1}$ were observed upon solvent exchange when compared with the as-prepared $1 \supset$ DMF (dimethylformamide); this is in agreement with a lower or higher number of intermolecular contacts, respectively. Flexibility of $\mathbf{1}$ \Guest is related to the flexible m-carborane bispyridylalcohol ligand (mCB) 
and to the supramolecular host-guest interactions, as demonstrated by the mechanical properties and crystalline sponge behavior of $\mathbf{1} \supset$ Guest.[16b] Here, we show that this SPC/MOF (1) shows an unprecedented reversible multi-step SCSC transformation from a 3D to a $2 \mathrm{D}$ framework through partial metal-linker bond cleavage/formation, rather than through the conventional metal-linker bond deformation.[1b] This reversible transformation is achieved by solvent-guest exchange under ambient conditions in one direction (3D to 2D) and in DMF solutions at $100{ }^{\circ} \mathrm{C}$ in the other (2D to $\left.3 \mathrm{D}\right)$. A combination of single-crystal (SCXRD), powder (PXRD) X-ray diffraction and DFT calculations provided an unusually detailed structural insight into the observed reversible dynamic behavior. Moreover, it is demonstrated that such reversible transformation can be used for entrapping molecular guests that are too large to diffuse into the initial 3D structure, disclosing a new approach for exploring encapsulation in other flexible MOFs. 


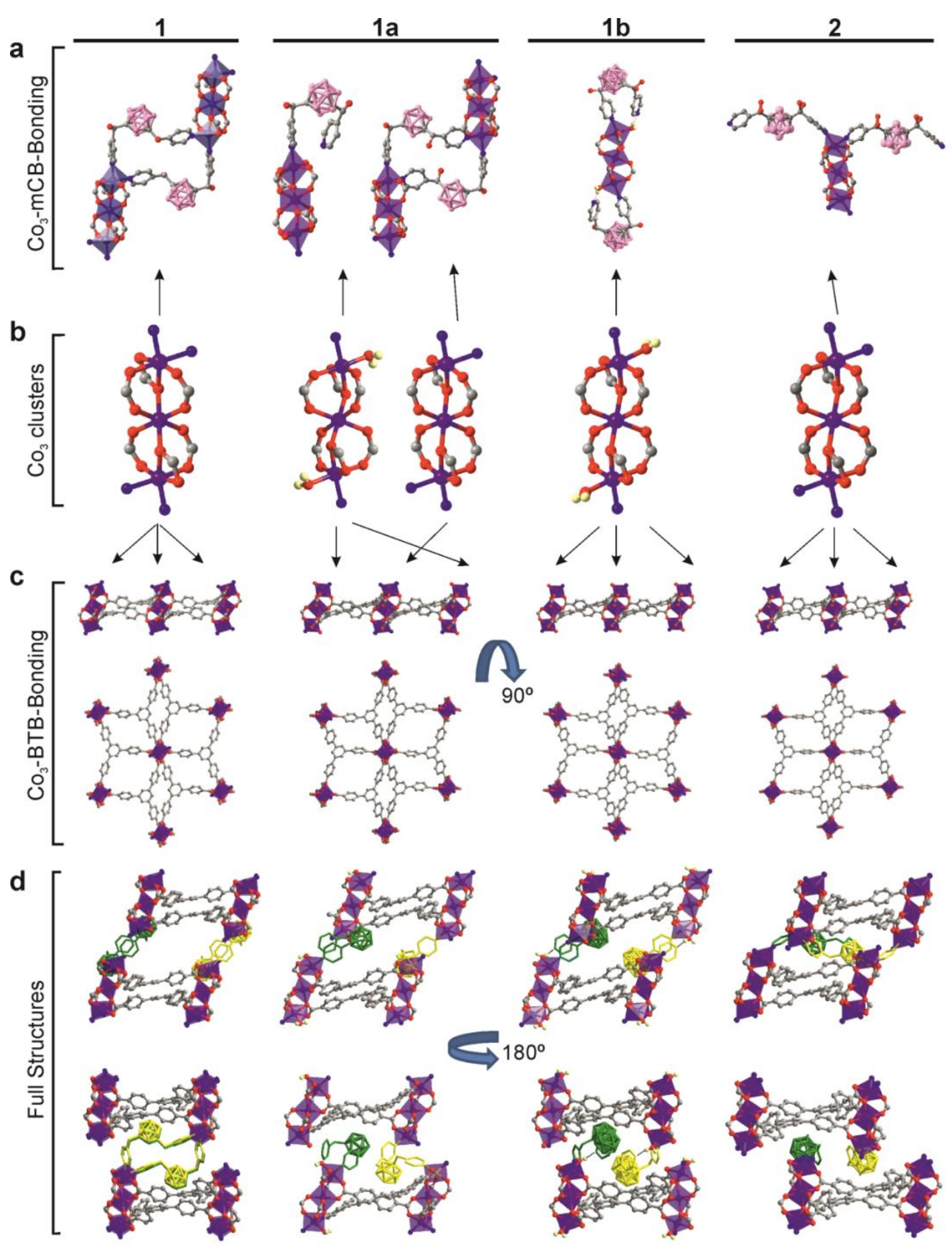

Figure 1. Crystal structures of $\mathbf{1}, \mathbf{1 a}, \mathbf{1 b}$ and 2. a) View of the trinuclear $\mathrm{Co}$ (II) units (as blueviolet polyhedrons) with $\mathrm{mCB}$ coordination. b) View of the trinuclear $\mathrm{Co}$ (II) unit and coordination environment of the Co(II) ions. c) Six-pointed star motif trinuclear Co(II)/BTB layers. d) A comparative view of the structures represented in three dimensions along two perpendicular directions; for clarity carborane linkers are here colored green or yellow and the $\mathrm{OH}$ groups are omitted. $\mathrm{H}$ atoms (except that for $\mathrm{H}_{2} \mathrm{O}$ ) are omitted for clarity. Color code: $\mathrm{H}$ yellow; B pink; $\mathrm{C}$ grey; $\mathrm{O}$ red; $\mathrm{N}$ blue, Co violet. 
The previously reported SPC [Co3(BTB $\left.)_{2}(\mathrm{mCB})_{2}\right] \cdot x \mathrm{DMF}(\mathbf{1} \supset \mathbf{D M F}$ or just $\mathbf{1})$ can be seen as a $2 \mathrm{D}$ rigid system composed of flat layers that can be opened and closed via flexible carborane linkers connecting the layers. 1 consists of trinuclear $\left[\mathrm{Co} 3(\mathrm{COO})_{6} \mathrm{~N}_{4}\right]$ units (left of Figure 1b), each coordinated to six different BTB ligands, thus creating six-pointed star motif $\mathrm{Co} / \mathrm{BTB}$ rigid layers extending in the $b c$ plane (left of Figure 1c). These layers are pillared by mCB ligands resulting in a 3D framework that exhibits 1D channels along the $b$ axis (Figure 1d and Figure S1, Supporting Information).[16b] The flexibility of the carborane ligands was evident by nanoindentation experiments, as the association of layers occurs through two carborane ligands that adopt an almost L-shape (left of Figures 1a and 1d). Thus, 1 can be seen as a model SPC with flexibility along one dimension, through the flexible ligands 3D aromatic based carborane system.

Immersion of crystals of $\mathbf{1} \supset$ DMF in methanol $(\mathrm{MeOH})$ for 1-2 days under ambient conditions showed a clear macroscopic transformation in the morphology of the crystals, from large rhombohedral crystals to thin plates (Supplementary Figure S2). Such a macroscopic change in the habit was also observed after a similar treatment in $\mathrm{CHCl}_{3}$ or by processing the crystals under a continuous flow of supercritical $\mathrm{CO}_{2}\left(\mathrm{scCO}_{2}\right)$ at $45^{\circ} \mathrm{C}$ and $20 \mathrm{MPa}$. No such changes were observed by immersion of $1 \supset$ DMF crystals in benzene, nitropropane, toluene or nitrobenzene or even in water for 2 days. Crystals of $\left[\mathrm{Co} 3(\mathrm{BTB})_{2}(\mathrm{mCB})_{2}\left(\mathrm{H}_{2} \mathrm{O}\right)\right] \cdot \mathrm{H}_{2} \mathrm{O}(\mathbf{1 a})$, $\left[\mathrm{Co} 3(\mathrm{BTB})_{2}(\mathrm{mCB})_{2}\left(\mathrm{H}_{2} \mathrm{O}\right)_{2}\right] \cdot x \mathrm{xMF}(\mathbf{1 b})$ and $\left[\mathrm{Co}_{3}(\mathrm{BTB})_{2}(\mathrm{mCB})_{2}\right] \cdot 4 \mathrm{H}_{2} \mathrm{O}(\mathbf{2})$, were recovered at different times of the solvent exchange process and their structures were solved by SCXRD. A full comparison of the structure of $\mathbf{1}$ with those obtained for $\mathbf{1 a}, \mathbf{1 b}$ and $\mathbf{2}$ is shown in Figure 1. Structural details are provided in the Supplementary. The basic unit of $\mathbf{1}, \mathbf{1 a}, \mathbf{1 b}$ and 2 is a trinuclear $\mathrm{Co}_{3}(\mathrm{COO})_{6}$ cluster in which the central Co atoms adopt an octahedral geometry $\left\{\mathrm{O}_{6}\right\}$-coordinated to six neighboring BTB ligands (Figure 2). The terminal Co 
atoms of the trinuclear $\mathrm{Co} 3(\mathrm{COO}) 6$ clusters also adopt an octahedral geometry, however, they differ in the nature of their terminal ligands and this has a pronounced effect on their 3D structures as explained below. In $\mathbf{1}$ and $\mathbf{2}$ both terminal Co atoms are $\left\{\mathrm{N}_{2} \mathrm{O}_{4}\right\}$-octahedrally coordinated to two mCB ligands and to four O atoms of three BTB ligands (Figure 2 and S3). In contrast, both terminal $\mathrm{Co}$ atoms in $\mathbf{1 b}$ are $\left\{\mathrm{NO}_{5}\right\}$-octahedrally coordinated to one $\mathrm{mCB}$ ligand, four $\mathrm{O}$ atoms of three $\mathrm{BTB}$ ligands and one $\mathrm{O}$ atom from a coordinated $\mathrm{H}_{2} \mathrm{O}$ molecule (Figure 1a-b). Interestingly, an uncoordinated pyridine moiety from the $\mathrm{mCB}$ ligand is $\mathrm{O}-$ $\mathrm{H}^{\cdots} \mathrm{N}$ hydrogen bonded to the coordinated $\mathrm{H}_{2} \mathrm{O}$ molecule in $\mathbf{1 b}\left(\mathrm{H}^{\cdots} \mathrm{N}, 1.83(3) \AA\right.$; $\mathrm{OHN}$, 172(4) ${ }^{\circ}$; Figure 1a and Supplementary Figure S4). The situation is more complex in the case of $\mathbf{1 a}$ as the terminal Co atoms alternate throughout the structure, either $\left\{\mathrm{N}_{2} \mathrm{O}_{4}\right\}-$ or $\left\{\mathrm{NO}_{5}\right\}-$ octahedral coordination (Figures 1a-b). As mentioned above, the Co/BTB layers are pillared by mCB ligands resulting in a 3D framework that exhibits $1 \mathrm{D}$ channels along the $b$ axis in $\mathbf{1}$ (Figures 1d and S1).[16b] That is, each pyridine moiety of one mCB ligand is coordinated to a terminal Co atom of contiguous Co/BTB layers. In $\mathbf{1 a}$ and $\mathbf{1 b}$, some or all of the mCB ligands are not acting as pillars (Figure 1a) since one of the two pyridyl moieties per mCB linker is no longer directly bonded to a Co atom. The Co-N bond cleavage generates unsaturated vacant sites at the metals that has been occupied by an $\mathrm{H}_{2} \mathrm{O}$ molecule, as exhibited in the crystal structures 1a and 1b (Figure 1). The consequence of such partial (1a) or total (1b) Co-N bond cleavage of one of the pyridyl moieties per $\mathrm{mCB}$ linker is the transformation of a 3D structure (1a) into a 2D structure (1b) with an associated loss of porosity (Figure 1d). Such a 2D structure is also observed in $\mathbf{2}$, however the uncoordinated pyridine moieties in $\mathbf{1 b}$ are now again coordinated to Co atoms (e.g. displacing the Co-coordinated $\mathrm{H}_{2} \mathrm{O}$ molecules in $\mathbf{1 b}$ ), but now to a contiguous trinuclear $\mathrm{Co} 3(\mathrm{COO})_{6}$ cluster in the same layer (Figures 1a and 1d). Figures 2 and S3 show a schematic representation of the transformation (see also Supplementary video). The SCXRD data confirms that a $3 \mathrm{D}(\mathbf{1}, \mathbf{1 a})$ to $2 \mathrm{D}(\mathbf{1 b}$ and $\mathbf{2})$ 
transformation is feasible by solvent exchange in $\mathrm{MeOH}, \mathrm{CHCl}_{3}$ and by desolvation aided by $\mathrm{scCO}_{2}$.

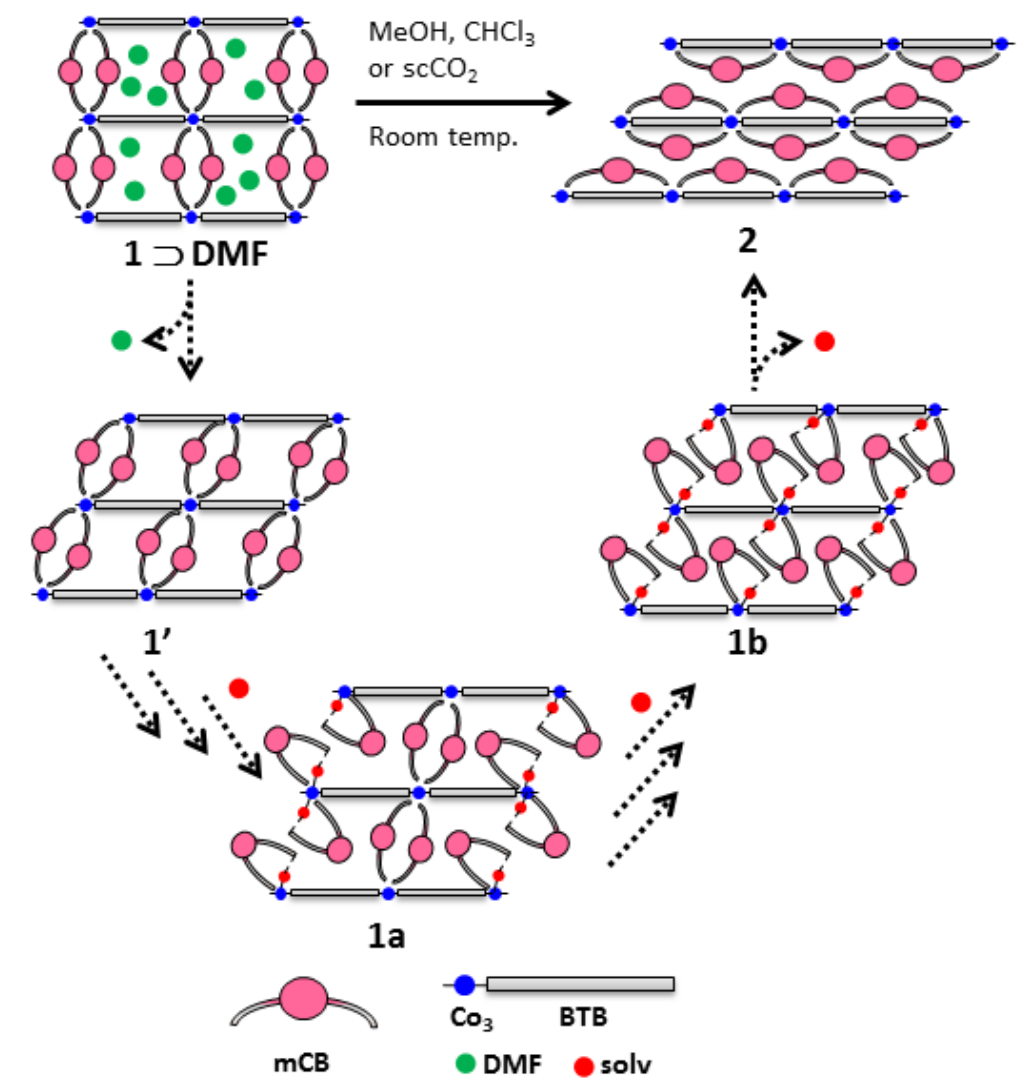

Figure 2. Schematic illustration of the multi-step SCSC transformations from 1 to 2 . Proposed mechanism for the 3D to $2 \mathrm{D}$ transformation based on the SCXRD analysis. See also supplementary video.

The observed arrangement of the $\mathrm{mCB}$ ligand during the transformation is certainly unusual: one of the pyridine moieties of each ligand uncoordinates from a Co center and coordinates to another Co in the same layer in a sort of inter-layer to intra-layer arrangement or folding of the mCB linkers (Figure 2). Such folding of the carborane linkers causes the "disconnection" of the $\mathrm{Co} / \mathrm{BTB}$ layer and therefore the 3D to $2 \mathrm{D}$ transformation (Figures 2 and $\mathrm{S} 3$ and Suplementary video). Structural conversion of compound 1 occurs when DMF guest molecules are exchanged with poor hydrogen bonding acceptors such as $\mathrm{MeOH}, \mathrm{CHCl}_{3}$ or $\mathrm{scCO}_{2}$ (vide infra). With the solvent-synthesis molecules gradually escaping, the unit cell parameters dramatically change while the space group symmetry is retained. Phase $\mathbf{2}$ was 
indexed with a cell similar to that of phase $\mathbf{1}$, except for a radically contracted $c$ axis (reduction of $38 \%$ ). In the absence of weak or specific host-guest interactions, the network contracts to fill the void space created by the displacement of DMF. The deformation of the network then provokes a displacement of the pyridyl ligands bound to the Co/BTB layers through a multi-step SCSC transformation to yield 2 (Figure 2). The process can be observed macroscopically as a change in the morphology and size of the crystals (Supplementary Figure S2). 1a-b are presumably not the only transition points between $\mathbf{1}$ and $\mathbf{2}$, but simply the ones that were characterized in this work, although other successive intermediates should be formed in a continuous structural transformation. Note that the single crystals used to elucidate 1a and $\mathbf{2}$ were obtained from the same sample, thus showing that the bulk crystals of 1 were not all transformed into 2 under the exchange conditions used. The isolation and characterization of these structural intermediates was crucial in proposing the mechanism for the dynamic transformation, since they provide convenient snapshots during the dynamic transformation.

Motivated by the above SCXRD results, we followed the transformation of 1 DMF crystals exposed to $\mathrm{MeOH}$ by powder X-ray diffraction (PXRD). Remarkably, the SCSC transformation from $\mathbf{1}$ to $\mathbf{2}$ could also be observed by PXRD (Figure S5, Supporting Information). Recorded profiles obtained by treating $\mathbf{1}$ in $\mathrm{MeOH}$ at different immersion times were compared with the calculated PXRDs patterns from single crystal data of structures $\mathbf{1}$ (Supplementary). The analysis of data reveals that $\mathbf{1}$ gradually transforms into $\mathbf{2}$ and other intermediate phases when exposed to $\mathrm{MeOH}$ for 2-5 days (Figure S5b-d). Crystals were then further treated at $40{ }^{\circ} \mathrm{C}$ under vacuum for $20 \mathrm{~h}$ (Figure S4e); however, complete transformation to phase 2 was only achieved after heating at $80^{\circ} \mathrm{C}$ (Figure S5f). The heating step is fully consistent with the above SCXRD results (Figure 2), as uncoordination of water (or any other solvent) from the cobalt centers in intermediate stages, such as e.g. 1b, is required in order to obtain 2. Hydration/dehydration of the $\mathrm{Co}(\mathrm{II})$ centers with 
displacement/coordination, respectively, of a 4,4'-bipyridine has been previously observed.[17] In addition to the PXRD data, unit cell determination by SCXRD of up to sixteen crystals of the transformed phase provides further support for the conversion of $\mathbf{1}$ to $\mathbf{2}$ (Supplementary Table S3). Furthermore, optimization of the framework transformation from $\mathbf{1}$ to $\mathbf{2}$ is achieved by solvent exchange of $\mathbf{1}$ DMF with $\mathrm{MeOH}$ or $\mathrm{CHCl}_{3}$ under ambient conditions for 5 days followed by heating at $80{ }^{\circ} \mathrm{C}$ under vacuum for $20 \mathrm{~h}$; or by straight treatment in a flow of $\mathrm{scCO}_{2}$ at $20 \mathrm{MPa}$ and $45{ }^{\circ} \mathrm{C}$ (Supplementary Figure S6 and Table S2). FT-IR, thermogravimetric analysis (TGA), elemental analysis and nitrogen sorption measurements (Supplementary Figures S7-8) of 2 confirms the non-porous nature of this phase, only containing residual water $(<5 \%)$.

Density Functional Theory (DFT) calculations were performed to analyze the energy contributions involved in the MOF transformation described in Figure 2. The structures of the systems $1 \supset$ DMF, 1' (1 with empty channels), 1b and $\mathbf{2}$ were thus optimized using the FHIaims code (PBE exchange correlation functional including dispersion energies, see Supplementary for details). Compound 1a was not considered due to its large unit cell. Energy comparison between 1 DMF and 1' clearly shows the large tendency to fill the channels of the latter with DMF molecules. The inclusion of DMF molecules in the 1' channels is strongly favored due to an interaction of $23.3 \mathrm{kcal} / \mathrm{mol}$ per solvent molecule while the reorganization energy of the host structure is only $10.0 \mathrm{kcal} / \mathrm{mol}$. The latter is the result of the presence of a large number of host-guest interactions $\left(\mathrm{O}-\mathrm{H} \cdots \mathrm{O}, \mathrm{C}-\mathrm{H} \cdots \mathrm{O}\right.$ and $\mathrm{C}-\mathrm{H}^{\cdots} \pi^{*}$; Supplementary). As previously reported,[16b] inclusion of DMF over $\mathrm{MeOH}$ is favored. The interaction of $\mathrm{MeOH}$ molecules with 1' is weaker than that for the DMF molecules, with an interaction energy of $9.1 \mathrm{kcal} / \mathrm{mol}$ per $\mathrm{MeOH}$ molecule and with a host reorganization energy of only $4.3 \mathrm{kcal} / \mathrm{mol}$. These results confirm the selectivity of the host structure towards DMF solvent. More importantly, an energy comparison between 1' (3D) and 2 (2D) reveals that the latter is $25.7 \mathrm{kcal} / \mathrm{mol}$ more stable than $\mathbf{1}^{\prime}$. It is worth noting that without dispersion 
contributions, $\mathbf{1}^{\prime}$ is $34.6 \mathrm{kcal} / \mathrm{mol}$ more stable than $\mathbf{2}$, showing the key role that inclusion of this term has in stabilizing $\mathbf{2}$ due to its compact structure. Finally, the optimized structure for the intermediate $\mathbf{1 b}$, containing four water and two DMF solvent molecules, shows relatively high stability. It is around $110.1 \mathrm{kcal} / \mathrm{mol}$ more stable than the 1' structure and a system containing six isolated solvent molecules (with the same structure as $\mathbf{1 b}$ ). Due to the presence of the host-solvent interaction in $\mathbf{1 b}$, such a system is also more stable than $\mathbf{2}(84.3 \mathrm{kcal} / \mathrm{mol}$, taking into account the energy of 2 plus the energy of the 4 water and 2 DMF solvent molecules set). In this case, the equilibrium is shifted towards the free solvent structure of 2 because the reaction is performed under vacuum and high temperature to facilitate the elimination of the solvent molecules as explained above. A detailed explanation of the procedure followed for the energy calculations can be found in the Supplementary.

The observed transformation involves the partial uncoordination of the mCB ligands and their re-coordination (folding linker) with simultaneous shifting of the Co/BTB layers in a very slow and concerted way, that maintains a significant degree of crystallinity for all intermediate phases involved in the transformation. Even if the crystallinity is not perfect, that just means that some long range order in the framework is lost, but without the collapse of the network.[4] Such a slow process could be due to the presence of the carborane moieties. The 3D globular shape of the carborane moieties could conceivably minimize the collapse of the structure when DMF is removed from the channels of $\mathbf{1} \supset$ DMF. For example, in the event of a porous pillared 3D structure collapse, the globular shaped pillars will help to maintain porosity as compared with the case of flat pillars. Another point to consider in the present transformation is the possible effect of water, as molecules of this solvent were positively identified in the solved structures of $\mathbf{1 a}, \mathbf{1 b}$ and $\mathbf{2}$. However, the water present in the structure, from the synthesis[18] or adsorbed from atmosphere during manipulation, does not seem to initiate the transformation, since the rate of the $3 \mathrm{D}$ to $2 \mathrm{D}$ transformation was not affected by the addition of water (up to $20 \mathrm{v} \%$ ) to the $\mathrm{MeOH}$ suspension. More importantly, 1 remains 
stable in water for weeks. The latter certainly indicates that water is not promoting the phase transformation. Thus, we believe that the displacement of DMF by poorer hydrogen bonding acceptor molecules (e.g., $\mathrm{MeOH}, \mathrm{CHCl}_{3}$ or $\mathrm{scCO}_{2}$ ) is the driving force for the transformation and this conclusion is clearly supported by the DFT calculations.

Formally, $\mathbf{1}$ and $\mathbf{2}$ are polymeric compounds with the same molecular formula. However the mCB linkers are folded into the Co/BTB layers in 2, but are unfolded in 1 (Figure 2). The higher calculated stability of $\mathbf{1} \supset \mathbf{D M F}$ as compared to $\mathbf{2}$ prompted us to consider whether the transformation could be reversible, that is, whether the folded $\mathrm{mCB}$ linkers in $\mathbf{2}$ can be unfolded to provide $\mathbf{1}$. Remarkably, this transformation of $\mathbf{1}$ into $\mathbf{2}$ is indeed reversible (Top of Figure 3). Crystals from 2 were converted into 1 by heating at $100{ }^{\circ} \mathrm{C}$ in DMF for $48 \mathrm{~h}$ and the transformation was confirmed by a combination of SCXRD and PXRD analysis (Supplementary). PXRD shows that the transformation of phase $\mathbf{2}$ into $\mathbf{1}$ took place under the above conditions, as indicated by the diffraction peak at $2 \Theta 6.4^{\circ}$ in the diffractogram, which only occurs for phase 1 (Supplementary Figure. S9). Further confirmation for the reversibility of this process was obtained from SCXRD performed on ten crystals of the bulk material recovered after the transformation (Supplementary Table S4). Such a flexible behavior, in which metal-linker bonds undergo cleavage in a reversible manner and without loss of the linkers, has not been previously described. There are only a few examples of metal-dipyridine bond cleavage, but with complete loss of the linkers, and therefore providing non reversible transformations.[19] In the present case, flexibility of the mCB ligand facilitates its partial decoordination (folding/unfolding) during the process and therefore reversibility is simply achieved by heating in DMF. This solvent is crucial for the reversibility process as no transformation was observed when using other poorly coordinating solvents such as acetonitrile or dichlorobenzene. 
a)

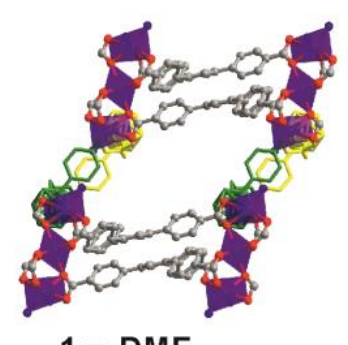

i) $\mathrm{MeOH}, \mathrm{CHCl}_{3}$ or $\mathrm{ScCO}_{2}$

ii) $80 \circ \mathrm{C}$ under vacuum

$1 \supset \mathrm{DMF}$
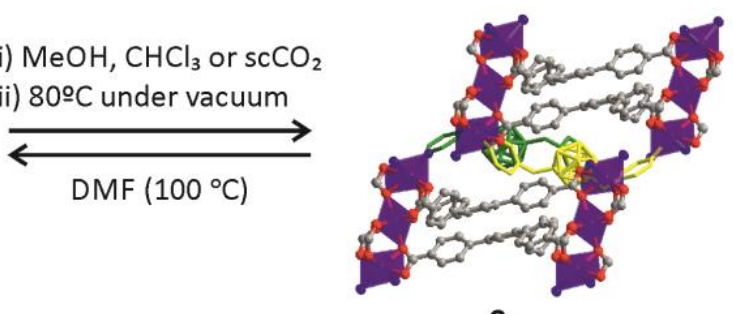

2

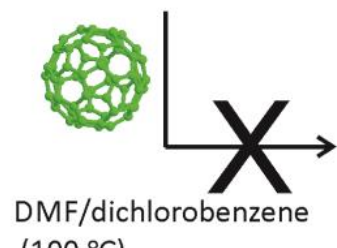

$\left(100^{\circ} \mathrm{C}\right)$
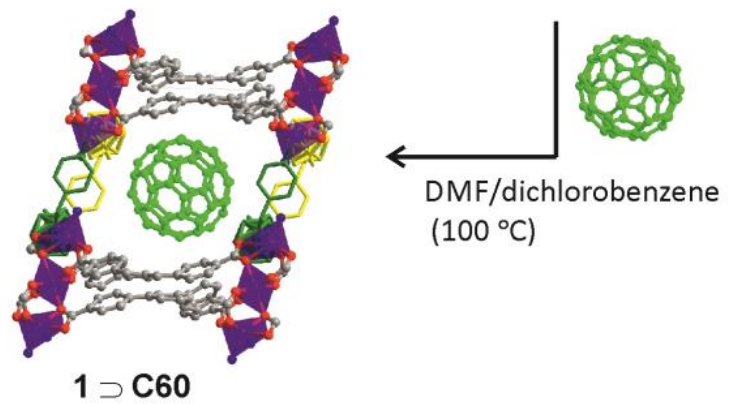

b)

i)

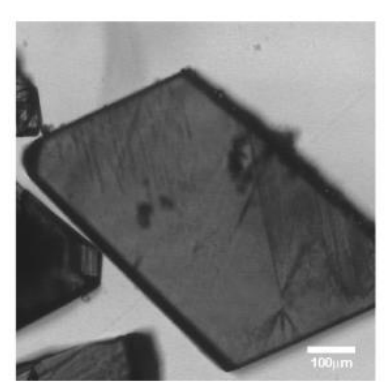

ii)

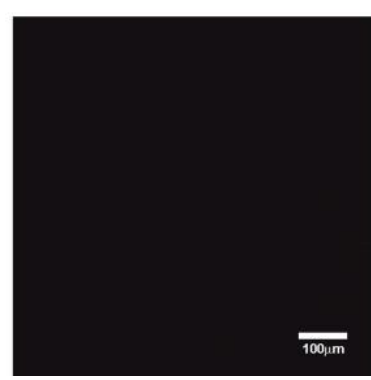

iii)

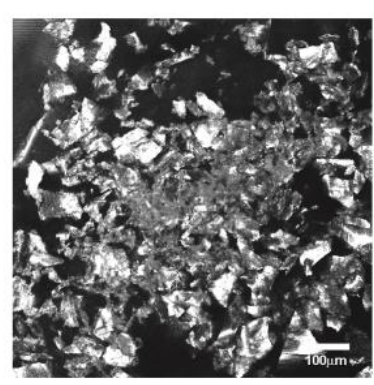

iv)

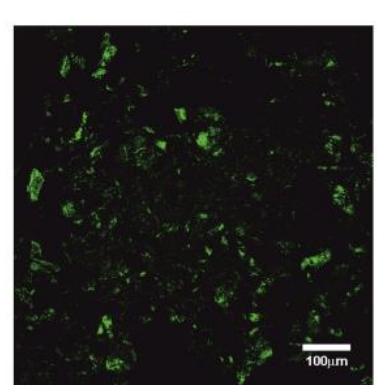

Figure 3. a) Optimized conditions for the bulk reversible $\mathbf{1}$ to $\mathbf{2}$ transformation and $\mathrm{C}_{60}$ encapsulation. $\mathbf{1} \supset \mathbf{D M F}$ and $\mathbf{2}$ are drawn from their crystal structures. $\mathbf{1} \supset \mathbf{C}_{60}$ is drawn from the DFT optimization. b) White light (top) and fluorescence images (bottom) for $\mathbf{1}$ and $\mathbf{1}$ C60 composites. i, white light transmission of 1. ii, fluorescence image of 1. iii, white light reflexion of $\mathbf{1} \supset \mathbf{C 6 0}$. iv, fluorescence image of $\mathbf{1} \supset \mathbf{C 6 0}$.

The reversibility of the $\mathbf{1}$ to $\mathbf{2}$ transformation opens a new way to encapsulate different guests. As previously mentioned, $\mathbf{1}$ behaves as a crystalline sponge for a variety of guests, mainly small organic compounds that stablish a large number of intermolecular interactions with the channel walls.[16b] Large channel dimensions of $\mathbf{1}(\sim 11 \times 13 \AA$ in $\mathbf{1} \supset \mathbf{D M F})$ are, in principle, suitable to accommodate bulky molecular compounds, such as e.g., [60]Fullerene (C60).[20] 
This is supported by DFT calculations ( $\mathbf{1} \supset \mathbf{C 6 0}$ in Figure 3a and Supplementary), which indicate that $\mathrm{C}_{60}$ inclusion energy on 1' is $60.0 \mathrm{kcal} / \mathrm{mol}$. This value can be split in two contributions, the host-guest interaction energy $(86.2 \mathrm{kcal} / \mathrm{mol})$, and a much smaller reorganization energy of the host contribution $(22.2 \mathrm{kcal} / \mathrm{mol})$. Conventional immersion experiments performed in concentrated $\mathrm{C}_{60}$ toluene solutions at $100{ }^{\circ} \mathrm{C}$ for one week or even direct synthesis of $\mathbf{1}$ in the presence of $\mathrm{C}_{60}$ proved ineffectual. Contrarily, $\mathrm{C} 60$ was trapped in $\mathbf{1}$ when the phase transformation $\mathbf{2} \rightarrow \mathbf{1}$ was performed in the presence of this fullerene (in a DMF/dichlorobenzene mixture, v/v = 3/1). PXRD characterization of the $\mathbf{1} \supset \mathbf{C} 60$ composite confirmed that the structure of phase $\mathbf{1}$ was essentially recovered upon $\mathrm{C}_{60}$ incorporation, since the signal at $6.4^{\circ}$ is clearly visible in the diffractogram (Supplementary, Figure S10). Proof for the formation of the $\mathbf{1} \supset \mathbf{C 6}$ composite was obtained by the combination of threedimensional confocal fluorescence, TGA and FT-Raman analysis (Supplementary). A bright green fluorescence was observed in $\mathbf{1} \supset \mathbf{C} 60$ crystals, from top surface downward to the median cross-section in every $1.5 \mu \mathrm{m}$ depth (Figure $3 \mathrm{~b}$ and Supplementary Figure S11), evidencing that C60 was homogeneously distributed throughout the MOF matrix.[21] In contrast, no fluorescence was observed in $\mathbf{1} \supset$ DMF or after incubating $\mathbf{1}$ in $\mathrm{C}_{60}$ DMF/dichlorobenzene solutions at $100{ }^{\circ} \mathrm{C}$ in DMF for $48 \mathrm{~h}$. A small broad band centered at $1461 \mathrm{~cm}-1$ in the FT-Raman spectrum of $\mathbf{1} \supset \mathrm{C}_{60}$ composites, corresponding to the $\operatorname{Ag}(2)$ mode of 1-trapped $\mathrm{C}_{60,[21]}$ add further evidence for its incorporation within 1 (Supplementary, Figure S13). The $\mathrm{C}_{60}$ content in $\mathbf{1} \supset \mathbf{C 6 0}$ composite was estimated by TGA as about 2 wt.\% (Supplementary, Figure S12).

In conclusion, a flexible carborane based dipyridine ligand (mCB) was employed as the linker in $\mathbf{1}$ to explore the framework flexibility and possible influence of the icosahedral core of the ligand on the stability of $\mathbf{1}$. We report that the $3 \mathrm{D} \operatorname{MOF}\left[\mathrm{Co}(\mathrm{BTB})_{2}(\mathrm{mCB})_{2}\right] \cdot \mathrm{xDMF}(\mathbf{1}$ 
DMF) shows a flexible and reversible dynamic behavior distinct from that previously reported for other porous materials. A solid phase transition can be induced in $\mathbf{1}$ to generate a new non porous $2 \mathrm{D}$ structure $\left[\mathrm{Co} 3(\mathrm{BTB})_{2}(\mathrm{mCB})_{2}\right] \cdot x \operatorname{Solv}(2)$ without loss of the carborane linkers, that are now coordinated to Co atoms in the same layer (linker folding). The structural transformation was visualized by snapshots of the multi-step SCSC transformations by single and powder XRD. Poor hydrogen bond acceptors such as $\mathrm{MeOH}, \mathrm{CHCl}_{3}$ or $\mathrm{scCO}_{2}$ induce such 3D to 2D transformation by exchange of the DMF in the channels of $\mathbf{1}$. In the absence of stabilizing DMF, the structure of $\mathbf{1}$ is not stable and suffers a solid phase transition leading to 2 as the most stable product. Gas phase calculation provides full support for the observed transformations. Remarkably, the transformation is reversible and $\mathbf{2}$ can be further converted back into 1 by just heating in DMF. Intra-layer Co-N bond cleavage (linker unfolding) and inter-layer Co-N formation occur under such conditions, with the recovery of the initial porous 3D structure. As a proof of concept for potential applications, encapsulation of $\mathrm{C}_{60}$ has been achieved by trapping during the $2 \mathrm{D}$ to $3 \mathrm{D}$ phase transition, whereas no adsorption was observed by diffusion on the pores of the $3 \mathrm{D}$ phase 1 . The observed process constitutes a new way to encapsulate large molecules that cannot easily diffuse into the porous material.

Given the versatile composition of MOFs, and the fact that they can be fabricated by design, we believe our work here will facilitate development of flexible MOFs with reversible breathing or phase transitions triggered by stimuli such as guest exchange or temperature. Other spherical and flexible ligands can be envisaged as key players to increase the stability of further flexible porous materials. Such materials would ultimately provide very exciting pathways for obtaining smart porous materials with application opportunities in, e.g. selective gas adsorption, chemical sensing or hazardous waste adsorption. 
[CCDC 1820704-1820706 contains the supplementary crystallographic data for this paper.

These data can be obtained free of charge from The Cambridge Crystallographic Data Centre via www.ccdc.cam.ac.uk/data_request/cif.]

\section{Supporting Information}

Supporting Information is available from the Wiley Online Library or from the author.

\section{Acknowledgements}

F. Tang., F. Teixidor, C.V. and J.G.P. thank MEC grants CTQ2013-44670-R and CTQ201675150-R and the Generalitat de Catalunya (2014/SGR/149) for financial support. C.D, A.L.P. and A.B. thank MEC grants CTQ2014-56324 and CTQ2017-83632 and the Generalitat de Catalunya with grant 2014SGR377. J.C. and E.R. acknowledge to MEC for the grant CTQ2015-64579-C3-1-P and the computer resources, technical expertise and assistance provided by the Barcelona Supercomputing Centre. E.R. thanks the Generalitat de Catalunya for an ICREA Academia award. ICMAB acknowledges the support of the Spanish MINECO through the Severo Ochoa Centers of Excellence Program, under Grant SEV-2015-0496. F. Tang is enrolled in the UAB PhD program and acknowledges the China Scholarship Council (CSC) for her $\mathrm{PhD}$ grants (201506060171). A.L.P. acknowledges the RyC-2012-11588 contract. We thank Prof. J. Sort for help with the high vacuum furnace. Some of the experiments were performed at the XALOC beamline of the ALBA synchrotron with the support of ALBA staff.

Received: ((will be filled in by the editorial staff)) Revised: ((will be filled in by the editorial staff)) Published online: ((will be filled in by the editorial staff)) 
Metal-organic frameworks (MOFs) (also known as porous coordination polymers) can exhibit a reversible porous 3D (1) to non-porous 2D (2) phase transition by metal-nitrogen bond breaking and reforming without loss of the critical carborane based linkers, owing to the spherical shape of the linker's core. The structural transformation, which was visualized by snapshots of the multi-step SCSC transformations, can be used for entrapping molecular guests that are too large to diffuse into the initial 3D structure.

metal-organic frameworks, soft porous crystals, flexible porous materials, carboranes

F. Tan, A. López-Periago, M. E. Light, J. Cirera, E. Ruiz, A. Borrás, F. Teixidor, C. Viñas, C. Domingo, J. G. Planas

An Unprecedented Stimuli Controlled Single-crystal Reversible Phase Transition of a Metal-Organic Framework and its Application to a Novel Method of Guest Encapsulation

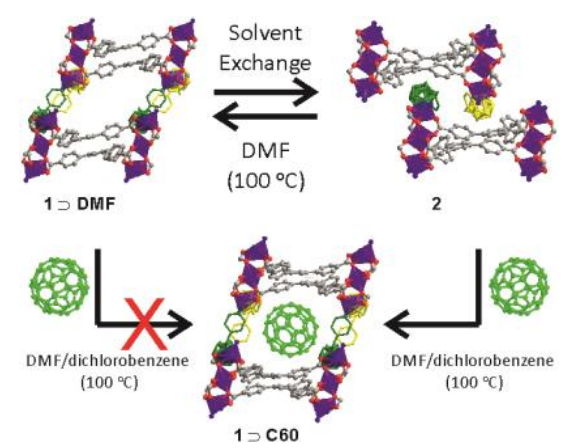


References

[1] a) X. Wang, C. Chi, K. Zhang, Y. Qian, K. M. Gupta, Z. Kang, J. Jiang, D. Zhao, Nature Communications 2017, 8, 14460; b) E. J. Carrington, C. A. McAnally, A. J. Fletcher, S. P. Thompson, M. Warren, L. Brammer, Nat Chem 2017, 9, 882-889; c) W. Cai, A. Gładysiak, M. Anioła, V. J. Smith, L. J. Barbour, A. Katrusiak, J. Am. Chem. Soc. 2015, 137, 9296-9301; d) A. B. Cairns, J. Catafesta, C. Levelut, J. Rouquette, A. Van Der Lee, L. Peters, A. L. Thompson, V. Dmitriev, J. Haines, A. L. Goodwin, Nature Materials 2013, 12, $212-$ 216; e) G. K. Kole, J. J. Vittal, Chem. Soc. Rev. 2013, 42, 1755-1775; f) J. Rabone, Y. F. Yue, S. Y. Chong, K. C. Stylianou, J. Bacsa, D. Bradshaw, G. R. Darling, N. G. Berry, Y. Z. Khimyak, A. Y. Ganin, P. Wiper, J. B. Claridge, M. J. Rosseinsky, Science 2010, 329, 1053 1057.

[2] S. S. Nagarkar, A. V. Desai, S. K. Ghosh, Chemistry - An Asian Journal 2014, 9, 2358-2376.

[3] a) J. R. Long, O. M. Yaghi, Chem. Soc. Rev. 2009, 38, 1213-1214; b) S. Kitagawa, R. Kitaura, S.-i. Noro, Angew. Chem. Int. Ed. 2004, 43, 2334-2375; c) S. L. James, Chem. Soc. Rev. 2003, 32, 276-288; d) H. Li, M. Eddaoudi, M. O'Keeffe, O. M. Yaghi, Nature 1999, 402, 276-279.

[4] S. Horike, S. Shimomura, S. Kitagawa, Nature Chemistry 2009, 1, 695-704.

[5] a) R. E. Morris, L. Brammer, Chem. Soc. Rev. 2017, 46, 5444-5462; b) G. Férey, New Journal of Chemistry 2016, 40, 3950-3967; c) C. R. Murdock, B. C. Hughes, Z. Lu, D. M. Jenkins, Coord. Chem. Rev. 2014, 258-259, 119-136; d) A. Schneemann, V. Bon, I. Schwedler, I. Senkovska, S. Kaskel, R. A. Fischer, Chem. Soc. Rev. 2014, 43, 6062-6096; e) Z.-J. Lin, J. Lu, M. Hong, R. Cao, Chem. Soc. Rev. 2014, 43, 5867-5895.

[6] a) N. A. Khan, Z. Hasan, S. H. Jhung, Journal of Hazardous Materials 2013, 244-245, 444-456; b) R. J. Kuppler, D. J. Timmons, Q. R. Fang, J. R. Li, T. A. Makal, M. D. Young, D. Yuan, D. Zhao, W. Zhuang, H. C. Zhou, Coord. Chem. Rev. 2009, 253, 3042-3066; c) J. R. Li, R. J. Kuppler, H. C. Zhou, Chem. Soc. Rev. 2009, 38, 1477-1504.

[7] a) G. Férey, Dalton Trans. 2016, 45, 4073-4089; b) B. Manna, A. V. Desai, S. K. Ghosh, Dalton Trans. 2016, 45, 4060-4072.

[8] a) F. A. Almeida Paz, J. Klinowski, S. M. F. Vilela, J. P. C. Tomé, J. A. S. Cavaleiro, J. Rocha, Chem. Soc. Rev. 2012, 41, 1088-1110; b) A. Y. Robin, K. M. Fromm, Coord. Chem. Rev. 2006, 250, 2127-2157.

[9] C. Martí-Gastaldo, D. Antypov, J. E. Warren, M. E. Briggs, P. A. Chater, P. V. Wiper, G. J. Miller, Y. Z. Khimyak, G. R. Darling, N. G. Berry, M. J. Rosseinsky, Nature Chemistry 2014, 6, 343.

[10] a) Y. Li, L. Gan, Chem. Eur. J. 2017, 23, 10485-10490; b) H. Prinzbach, F. Wahl, A. Weiler, P. Landenberger, J. Wörth, L. T. Scott, M. Gelmont, D. Olevano, F. Sommer, B. Von Issendorff, Chem. Eur. J. 2006, 12, 6268-6280; c) R. J. Ternansky, D. W. Balogh, L. A. Paquette, J. Am. Chem. Soc. 1982, 104, 4503-4504.

[11] a) J. Poater, M. Solà, C. Viñas, F. Teixidor, Chem. Eur. J. 2016, 22, 7437-7443; b) J. Poater, M. Solà, C. Viñas, F. Teixidor, Angew. Chem. Int. Ed. 2014, 53, 12191-12195.

[12] a) R. N. Grimes, in Carboranes (Third Edition), Academic Press, Oxford, 2016; b) M. Scholz, E. Hey-Hawkins, Chem. Rev. 2011, 111, 7035-7062; c) F. Teixidor, C. Viñas, in Science of Synthesis, Vol. 6 (Ed.: K. D.E.), 2005, p. 1325.

[13] a) R. N. Grimes, Dalton Trans. 2015, 44, 5939-5956; b) D. Olid, R. Núñez, C. Viñas, F. Teixidor, Chem. Soc. Rev. 2013, 42, 3318-3336.

[14] A. M. Cioran, A. D. Musteti, F. Teixidor, Z. Krpetić, I. A. Prior, Q. He, C. J. Kiely, M. Brust, C. Viñas, J. Am. Chem. Soc. 2012, 134, 212-221. 
[15] A. Saha, E. Oleshkevich, C. Vinas, F. Teixidor, Advanced Materials 2017.

[16] a) S. Rodriguez-Hermida, M. Y. Tsang, C. Vignatti, K. C. Stylianou, V. Guillerm, J. Perez-Carvajal, F. Teixidor, C. Vinas, D. Choquesillo-Lazarte, C. Verdugo-Escamilla, I. Peral, J. Juanhuix, A. Verdaguer, I. Imaz, D. Maspoch, J. Giner Planas, Angew Chem Int Ed Engl 2016, 55, 16049-16053; b) M. Y. Tsang, S. Rodríguez-Hermida, K. C. Stylianou, F. Tan, D. Negi, F. Teixidor, C. Viñas, D. Choquesillo-Lazarte, C. Verdugo-Escamilla, M. Guerrero, J. Sort, J. Juanhuix, D. Maspoch, J. Giner Planas, Cryst. Growth Des. 2017, 17, 846-857.

[17] D. Bradshaw, J. E. Warren, M. J. Rosseinsky, Science 2007, 315, 977-980.

[18] Combined TGA, IR and elemental analysis confirms the presence of $<3 \%$ water in the as made 1 (see ESI for full data).

[19] a) M. J. Cliffe, E. Castillo-Martínez, Y. Wu, J. Lee, A. C. Forse, F. C. N. Firth, P. Z. Moghadam, D. Fairen-Jimenez, M. W. Gaultois, J. A. Hill, O. V. Magdysyuk, B. Slater, A. L. Goodwin, C. P. Grey, J. Am. Chem. Soc. 2017, 139, 5397-5404; b) Z. Chen, S. Xiang, D. Zhao, B. Chen, Cryst. Growth Des. 2009, 9, 5293-5296.

[20] C. X. Yang, X. P. Yan, Journal of Materials Chemistry 2012, 22, 17833-17841.

[21] H. Li, M. R. Hill, R. Huang, C. Doblin, S. Lim, A. J. Hill, R. Babarao, P. Falcaro, Chem. Commun. 2016, 52, 5973-5976. 